\title{
Application Research of Ensemble Learning Algorithm in Image Annotation
}

\author{
Zhenxiang Lin $^{1, a}$, Jinlin Guo ${ }^{1, b}$, Songyang Lao ${ }^{1, c}$ \\ ${ }^{1}$ College of Information System and Management, National University of Defense Technology, \\ Changsha, 410005, China
}

Keywords: Ensemble learning algorithm, Image annotation, Machine learning

\begin{abstract}
Automatic image annotation is a fundamental and challenging task in the field of image retrieval. This paper introduces the concept and process of random forest algorithm. According to the characteristics of the current annotation model, the random forest algorithm is applied in image annotation field. The experimental results show that the machine learning algorithm used in this paper is more effective than traditional algorithms, which has a higher accuracy and a short processing time.
\end{abstract}

\section{Introduction}

With the development of digital multimedia technology and network bandwidth increase, all kinds of multimedia data such as image and video information has gradually become the mainstream of rapid expansion, and has a great impact on people's life and social development. Semantic clarity is an important prerequisite for large-scale multimedia data management, so it is of great theoretical and practical significance to study the semantic content of multimedia data objects automatically. Is the basis of the video image, plays an important role in the management of multimedia data, so the image semantic automatic annotation technology is currently a hot research field, caused great concern in academic and industrial circles. The aim of image annotation is to obtain the semantic key words according to the visual content of the image. Image semantic annotation usually includes two stages: model training and annotation. In the model training phase, the annotation model is learned from the given training set, and the new image is submitted by the user in the annotation stage. The performance of image annotation is usually related to the following two aspects: the description of the visual features of the image and the accuracy of the annotation model. The visual features of images are the basis of image annotation, and the extracted visual features should represent different visual attributes. The annotation model is the core of the image annotation, and the accuracy of annotation is closely related to the accuracy of the training. However, it is very difficult to learn from the limited training set to represent the real image and the real relationship of the text. It is an important content of image semantic annotation to find the appropriate model.

\section{Ensemble Learning Algorithm and Random Forest Algorithm}

Ensemble Learning Algorithm. Ensemble learning is a kind of machine learning method, which uses a series of learners to learn, and uses some rules to integrate the learning results to obtain a better learning effect than a single learner. Machine learning methods are widely used in production, research and life, and ensemble learning is the most popular direction of machine learning. Ensemble learning is a kind of machine learning method, which uses a series of learners to learn, and uses some rules to integrate the learning results to obtain a better learning effect than a single learner. The idea is to integrate learning when classifying new instances, a plurality of single classifier, through the classification of multi classifier results are some combination to determine the final classification, in order to obtain better performance than a single classifier. If a single classifier is compared to a decision maker, the ensemble learning approach is equivalent to the decision making process of multiple decision makers. The key to the success of integrated learning is to do everything possible to study the differences and intensity of learning. There are very close and difficult to describe the 
relationship between the two, there are opposites, there are synergies, we should balance the actual application of each other. The most important thing is to adjust the proportion of parameters according to different issues. When the feature correlation is too strong, the ensemble learning algorithm will adjust the proportion of random selection of features; when the sample noise is too large, the ensemble learning algorithm will adjust the proportion of randomly selected samples. Ensemble learning is a kind of machine learning method, which uses a series of learners to learn, and uses some rules to integrate the learning results to obtain a better learning effect than a single learner.

Random Forest Algorithm. Random forest is a classifier that uses multiple trees to train and predict samples. The classifier was first proposed by Leo Breiman and Adele Cutler, and was registered as a trademark. Classifier is given a sample of data to determine which category of the sample algorithm. For example, in the prediction of stock price, we believe that the trading volume of the day before and the closing price is for second days or so, the classifier is through the sample volume and the closing price forecast price second day's algorithm. In the process of decision tree training, it is necessary to divide the training data set into two subsets. In the classification problem, the data entered into the classifier is called the feature. To the above stock price forecast problem, for example, the characteristics of the previous day's trading volume and closing price. In the process of constructing a decision tree, we need to select the features from the whole sequence according to a certain order. A selected feature is a collection of features that have not been selected before the current step. Decision tree is a kind of basic classifier, which is usually divided into two categories. The constructed decision tree is a tree structure, which can be regarded as a set of if-then rules. We show the construction of decision tree by using the process image of the quantitative tool. If you want to choose a good quantitative tools to help us better stocks. Testing tools to provide data is not very comprehensive, the data is not comprehensive. A detection tool provides whether the API is easy to use. Test tool back to the test process is not reliable. It cannot fly back to the test strategy cannot use ah. Detection tool support does not support the simulation of the transaction. The light back only allows you to determine the history of the strategy is not useful, at least before the formal operation of a simulation disk. In this way, by whether the data is comprehensive, API is easy to use, back to test whether it is reliable, whether to support the simulation transactions will be quantified on the market tools affixed to labels.

\section{Image Annotation Based on Random Forest Algorithm}

The automatic image process based on random forest algorithm is shown in Figure 1 and Figure 2, which is divided into two steps. They are training and automatic semantic annotation. Firstly, each image in the training set segmentation of the image into a certain visual features of each region similarity, extraction of low-level visual features of each region, features preprocessing, produce different training set using the Bagging method, is used to generate each component classifier, finally by simple majority vote on the final classification result at the same time using manually labeled the annotation vocabulary and established the relationship with regional characteristics. Although the current image segmentation technology is not perfect, but it has been able to provide information based on visual features of each region in the image. For the segmented regions, they have approximately the same visual features, and can be described by a set of vectors that represent the underlying features of color and texture. Automatic image annotation is to find the best predefined text keywords to describe this set of eigenvalues. This set of feature values is mapped to a specific region, so that the image annotation problem is converted into the problem of automatic classification of images. Secondly, it is automatic semantic annotation. Each image is to be labeled, and then the visual features of the region to be tested are extracted. Similar data sets were randomly selected, every sub-tree split in random forest did not use all of the features to be selected, but from all the candidate features in randomly selected features, then feature in the feature selected randomly in the selection of the optimum. In this way, the decision trees in the random forest can be different from each other, so as to improve the diversity of the system and improve the classification performance. It will lead to focus on in the attribute, many irrelevant attributes, classification of strength will 
ultimately reduce the decision tree, increasing the generalization error bounds, and random forest accuracy. At this point, if we want to control the classification error rate, we need to increase the number of attributes extracted in each node, so as to extract more useful attributes, which will bring a large computational overhead. Finally, we use the classification model and classification rules in the training samples to classify the labeled regions, and to achieve the automatic image annotation.

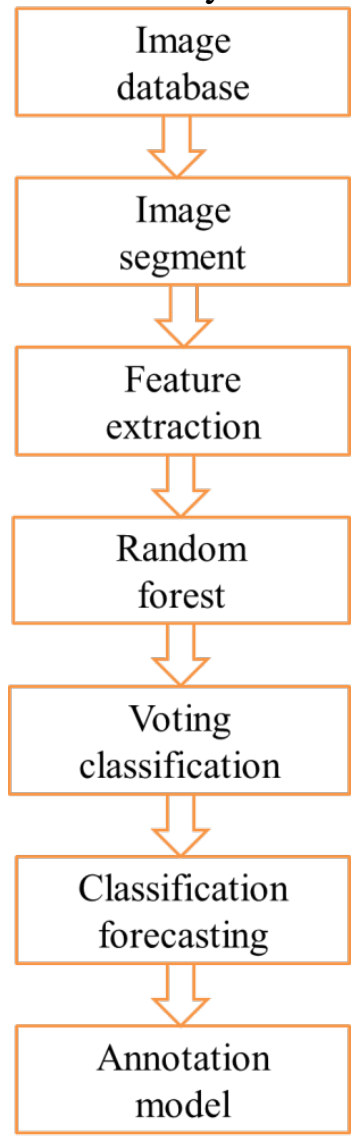

Figure 1. Figure of annotation model training annotation

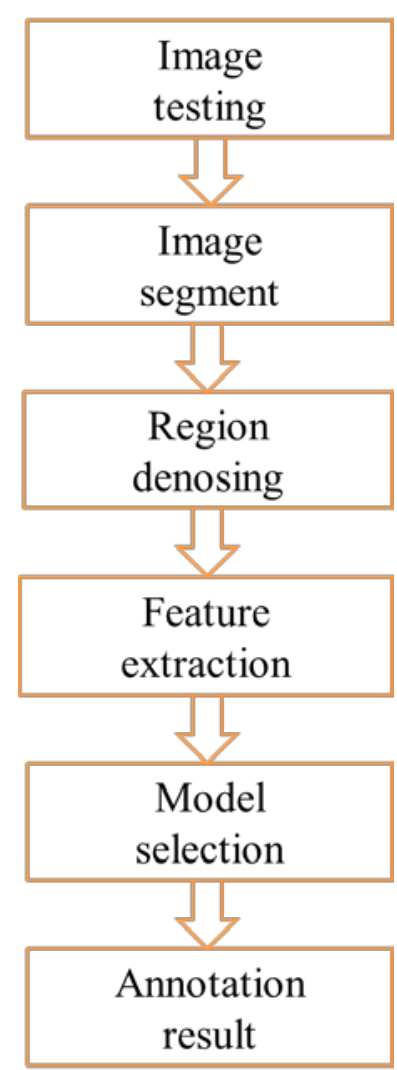

Figure 2. Figure of automatic semantic

\section{Results of Image Annotation Based on Random Forest Algorithm}

We use different algorithms for image annotation, compare the results. Common machine learning algorithms include C4.5, NewNBtree, SimpleC4.5, SVM, NB, KNN and RF. The classification accuracy and classification time as shown in Figure 2 and Figure 3:

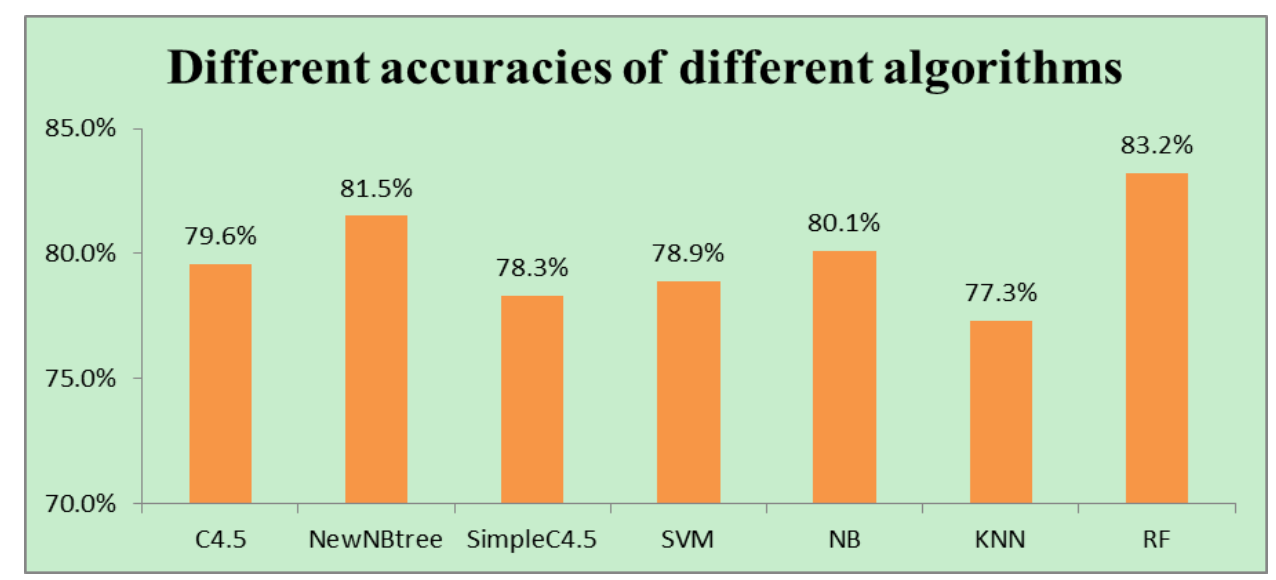

Figure 3. Different accuracies of different algorithms 


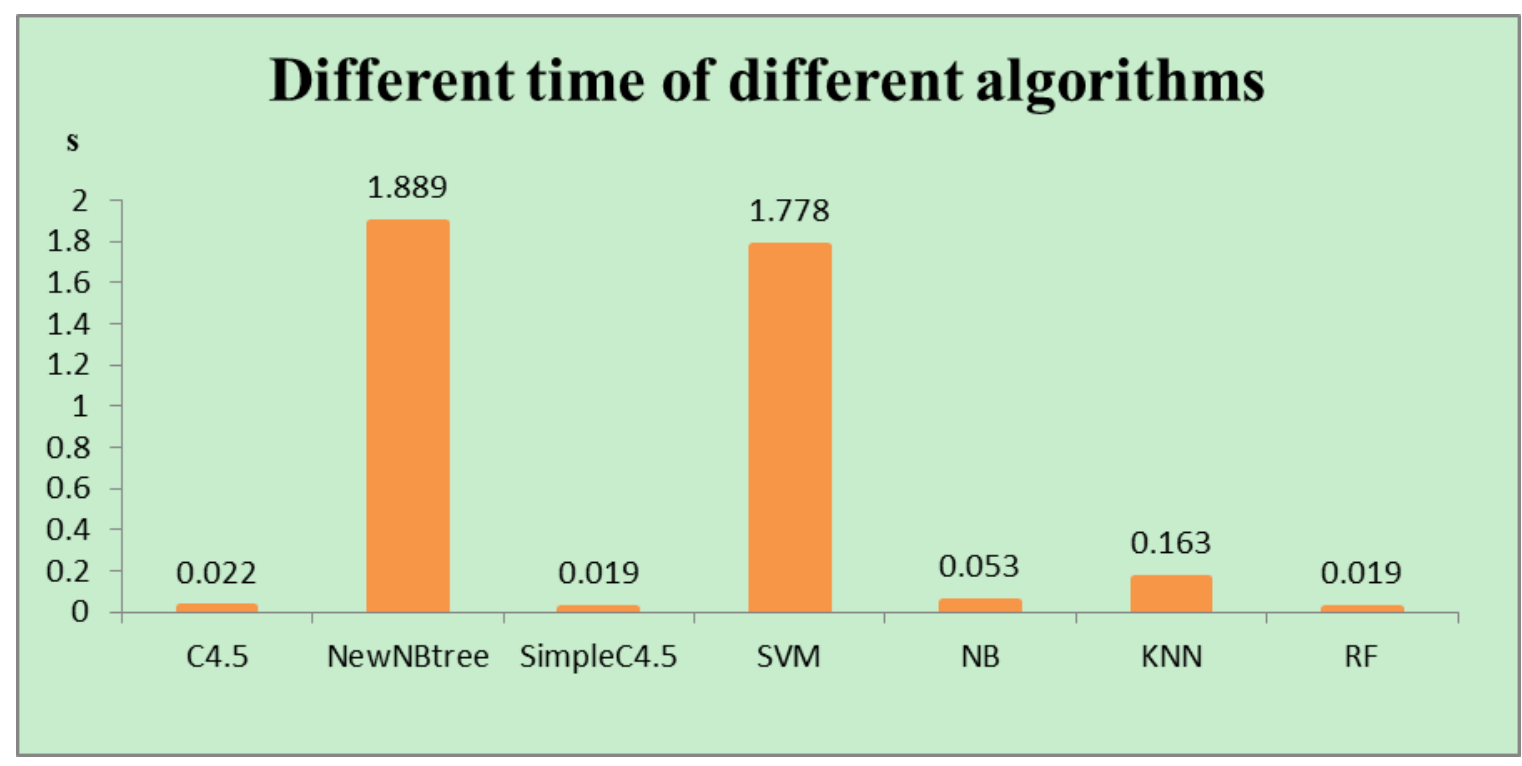

Figure 4. Different time of different algorithms

\section{Conclusion}

In this paper, an ensemble classification algorithm is used for automatic image annotation. This paper describes the process of annotation model training and automatic semantic annotation. The experimental results show that the algorithm is robust. Compared with other algorithms, the accuracy and speed of random forest algorithm are at the forefront, which is worthy of application and popularization.

\section{References}

[1] Yang Yang, Zhang Wensheng. Image Auto-Annotation Based on Deep Learning [J]. Journal of Data Acquisition and Processing, 2015, 30(1): 88-98.

[2] Zhang Huazhong, Hou Jin, Qin Xue, Xiao Ran. The Naive Bayes Classifier Ensemble Method and Its Application in Image Automatic Annotation [J]. Journal of Southwest University of Science and Technology, 2011, 26(3): 53-58.

[3] Jiang Lixing, Hou Jin. Image Annotation Using the Ensemble Learning [J]. Acta Automatica Sinica, 2012, 38(8):1257-1262.

[4] Luo Huilan, Wang Wei, Wang Hui. An image segmentation algorithm based on ensemble learning [J]. Journal of Jiangxi University of Science and Technology, 2012, 33(3): 56-61. 